\title{
Role of Coverage and Vacancy Defect in Adsorption and Desorption of Benzene on Si(001)-2×n Surface
}

\author{
Seung Chul Oh, Ki Wan Kim, Abdulla H. Mamun, Ha-Jin Lee, ${ }^{\dagger}$ and Jae Rayng Hahn* \\ Department of Chemistry and Institute of Photonics and Information Technology, \\ Chonbuk National University, Jeonju 561-756, Korea. ${ }^{*}$ E-mail: jrhahn@jbnu.ac.kr \\ $\dagger$ Jeonju Center, Korea Basic Science Institute, Jeonju 561-756, Korea \\ Received September 29, 2009, Accepted December 3, 2009
}

\begin{abstract}
We investigated the adsorption and desorption characteristics of benzene molecules on $\mathrm{Si}(001)-2 \times \mathrm{n}$ surfaces using a variable-low temperature scanning tunneling microscopy. When benzene was adsorbed on a $\mathrm{Si}(001)-2 \times \mathrm{n}$ surface at a low coverage, five distinct adsorption configurations were found: tight-binding (TB), standard-butterfly (SB), twistedbridge, diagonal-bridge, and pedestal. The TB and SB configurations were the most dominant ones and could be reversibly interconverted, diffused, and desorbed by applying an electric field between the tip and the surface. The population ratios of the TB and SB configurations were affected by the benzene coverage: at high coverage, the population ratio of SB increased over that of TB, which was favored at low coverage. The desorption yield decreased with increasing benzene coverage and/or density of vacancy defect. These results suggest that the interaction between the benzene molecules is important at a high coverage, and that the vacancy defects modify the adsorption and desorption energies of the benzene molecules on $\mathrm{Si}(001)$ surface.
\end{abstract}

Key Words: Scanning tunneling microscopy, Silicon surfaces, Benzene, Intermolecular interaction, Vacancy defect

\section{Introduction}

Research into the attachment of organic molecules to Si surfaces ${ }^{1}$ has intensified because of the potential applications of these systems in the construction of organic-silicon hybrid nanostructures for use in advanced molecular electronics, biosensors, and optical devices. For this reason, the chemisorption of organic molecules on Si surfaces has been examined in numerous theoretical and experimental investigations. In the construction of organic-silicon hybrids, molecular coverage on the Si surface is one of the crucial factors determining electronic device functions. Electronic property of organic molecular film devices varies with molecular packing pattern and the electric carrier mobility. In addition, vacancy defects on the Si surface should play important roles in determining the quality and function of the organic molecular devices, because they can be more reactive than clean $\mathrm{Si}$ or may determine the whole surface morphology of the Si surface. ${ }^{2}$ For more realistic applications, knowledge of the binding structures of organic molecules on $\mathrm{Si}$ surfaces as a function of coverage and defect density is therefore important to develop organic hybrid nanostructures.

The adsorption of benzene on Si substrates is of great interest as a model system for studying the molecular adsorption of hydrocarbons on semiconductor surfaces and because this system is considered as a promising precursor for technologically relevant processes, such as the chemical vapor deposition of diamond films on Si surfaces. Many experimental and theoretical studies have been reported on the adsorption mechanism of benzene on $\mathrm{Si}(001),{ }^{3-17} \mathrm{Si}(111),{ }^{18}$ and $\mathrm{Si}(5512)^{19}$ surfaces. While no complete experimental determinations have been made of the local chemisorption structure of benzene on $\mathrm{Si}(001)$, a number of different geometries have been proposed by a variety of techniques including thermal desorption spectroscopy (TDS), ${ }^{4,5}$ high-resolution electron energy loss spectroscopy, ${ }^{4}$ ultraviolet photoelectron spectroscopy (UPS), ${ }^{5}$ near-edge x-ray absorption fine structure (NEXAFS), ${ }^{6}$ high resolution photoemission spectroscopy, ${ }^{7}$ and scanning tunneling microscopy (STM) ${ }^{8,9,13-17}$ Several studies using STM and theoretical calculations $^{8-10}$ claim that benzene on $\operatorname{Si}(001)-2 \times 1$ is initially adsorbed in the standard-butterfly (SB) configuration and then quickly adopts the tight-binding (TB) configuration, which is more energetically stable. Taking into account the reported activation energies for this conversion-and the relative stabilities of the two configurations-complete conversion to the TB configuration would be expected relatively quickly. However, other studies using NEXAFS and UPS concluded that only the SB configuration is present on the surface as a major and stable species. ${ }^{11}$ A different, first-principles calculations based on cluster models supported this conclusion. ${ }^{12}$ Many aspects of the local structure of adsorbed benzene on $\mathrm{Si}(001)-2 \times 1$ at high coverage also remain unclear.

Such discrepancies can be due to two possibilities. (i) The STM and conventional ensemble-averaged spectroscopy studies focused on the very initial and the saturated coverage, respectively. (ii) The STM studies must be conducted at very low defect density while the defect density is hardly defined on the ensemble-averaged spectroscopic studies. Therefore, the coverage- and/or defect-dependent change of the adsorption characteristics observed here may reconcile the apparent discrepancies between the previous studies. On the $\mathrm{Si}(001)-2 \times$ n surface, we found five types of adsorption configurations of benzene molecules. Their relative population ratios, as well as the intermolecular-conversion and desorption processes, were studied using STM. These characteristics were found to differ from those of 
the $\mathrm{Si}(001)-2 \times 1$ surface. The population ratio changed upon increasing the benzene coverage, and the desorption yield decreased with increasing benzene coverage and defect density.

\section{Experimental}

When a $\operatorname{Si}(001)$ substrate is contaminated with a very small amount of $\mathrm{Ni}$, it is reconstructed to a $2 \times \mathrm{n}$ structure, ${ }^{2}$ which has many di-vacancy defects and short $\mathrm{Si}$-dimer rows. The $\mathrm{Si}(001)$ samples employed in this work were slightly contacted with a Ni rod and then ultrasonicated with ethyl alcohol. After degassing overnight at $700{ }^{\circ} \mathrm{C}$ in a UHV system (with a pressure below $1 \times 10^{-10}$ Torr), clean Si samples were prepared by repeated cycles of $500 \mathrm{eV} \mathrm{Ne}{ }^{+}$-ion sputtering and flashing at $1450 \mathrm{~K}$. We found that excessive surface roughening occurred if the vacuum pressure exceeded $3 \times 10^{-9}$ Torr during flashing at 1450 $\mathrm{K}$. To maintain the pressure below $3 \times 10^{-9}$ Torr during flashing, the heater and the sample holder had to be heavily degassed before flashing. After flashing, the sample was rapidly cooled to $1200 \mathrm{~K}$, and then to room temperature at a rate of $2 \mathrm{~K} / \mathrm{s}$ for surface reconstruction. The cleanliness of the samples was confirmed using our home-made, variable-low temperature STM equipment. ${ }^{20,21}$ The base pressure of the vacuum chamber was below $2 \times 10^{-11}$ Torr. Benzene (Aldrich) was purified by several freeze-pump-thaw cycles before being dosed onto the Si surface at room temperature through a leak valve equipped with a microcapillary-array-filled tube for uniform adsorption on the surface. The use of the microcapillary-array doser minimized the increase of chamber background pressure during dosing. STM characterizations were performed either at room or low temperatures. Electrochemically etched tungsten tips were prepared in the heater by means of repeated cycles of self-sputtering by field emission in a $\mathrm{Ne}$ atmosphere and heating in a strong electric field.

\section{Results and Discussion}

Adsorption configurations of benzene. Benzene molecules were adsorbed on the $\mathrm{Si}(001)-2 \times \mathrm{n}$ surface at room temperature, and the resulting surface was investigated with STM. Figure 1 shows representative STM topographical images (filled-state) obtained after benzene adsorption. By comparing this image with that of a clean surface, the several features in the image were attributed to adsorbed benzene molecules. The depression features (red) indicate Si vacancy defects. The benzene features can be classified into five different configurations according to their appearance, labeled $\boldsymbol{a}$ to $\boldsymbol{e}$. The number of features increased with the exposure to benzene, which verified that they correspond to adsorbed molecules. The benzene features appeared mainly along the Si-dimer rows, and no preferential adsorption of benzene molecules was observed on the defects. Figure 2 shows magnified STM images of benzene features. Feature $\boldsymbol{a}$ appeared as symmetrically elongated protrusions along the [110] direction in the filled-state image. Feature $\boldsymbol{a}$ was the highest protrusion, being slightly higher than feature $\boldsymbol{b}$ in the topographical image. Feature $\boldsymbol{b}$ appeared as asymmetrically elongated protrusions along the [110] direction. In the emptystate image (not shown), these features also appeared as protru-

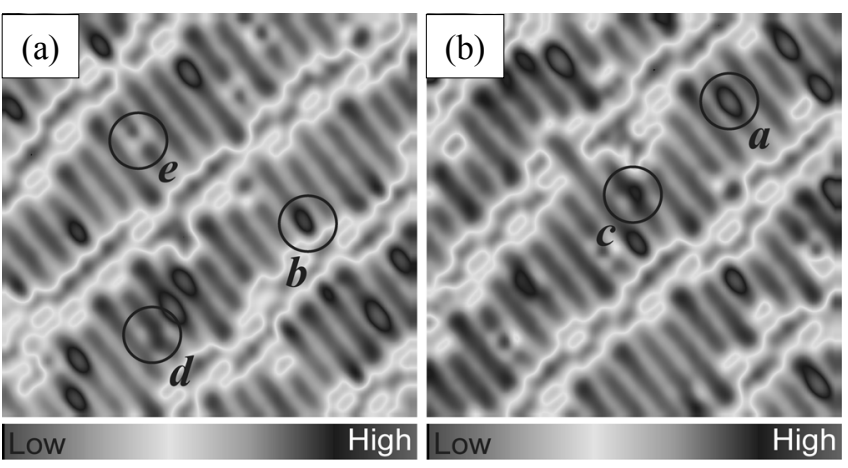

Figure 1. STM topographical images of benzene molecules adsorbed on $\operatorname{Si}(001)-2 \times$ n. Images (a) and (b) show different areas of the same surface. The images were obtained at a sample bias of $-1.5 \mathrm{~V}$ and a tunneling current of $0.5 \mathrm{nA}$. Five types of features (i.e., $\boldsymbol{a}, \boldsymbol{b}, \boldsymbol{c}, \boldsymbol{d}$, and $\boldsymbol{e}$ ) are observed in the images.
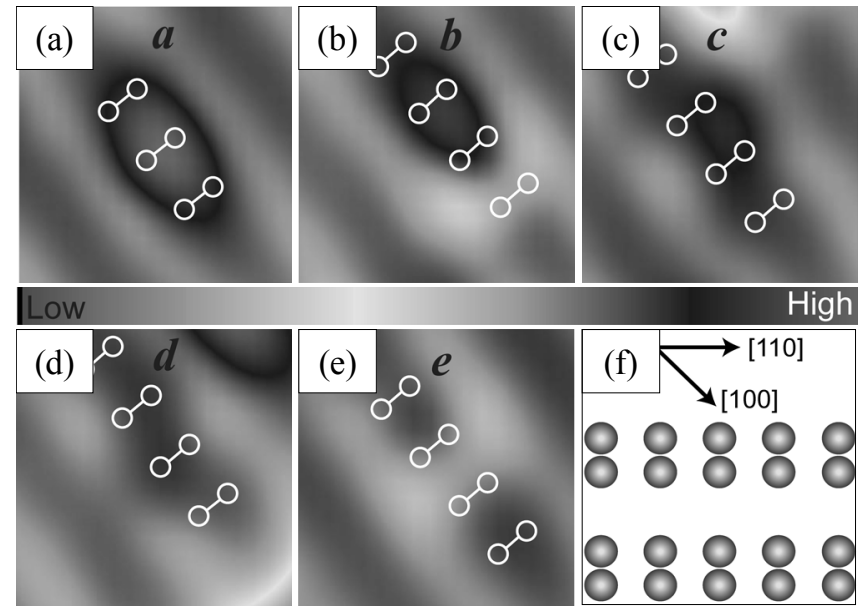

Figure 2. (a) (e) Magnified STM images of features $\boldsymbol{a}, \boldsymbol{b}, \boldsymbol{c}, \boldsymbol{d}$, and $\boldsymbol{e}$ in Fig. 1. Lines and circles in images indicate $\mathrm{Si}$ substrate structures. (f) The arrangement of Si dimers in the $2 \times 1$ reconstruction of $\mathrm{Si}(001)$. The up- and down-pattern of Si dimer atoms is not included.

sions, but the elongation was slightly reduced. Moreover, the features corresponding to $\boldsymbol{c}, \boldsymbol{d}$, and $\boldsymbol{e}$ were lower than those corresponding to features $\boldsymbol{a}$ and $\boldsymbol{b}$. Features $\boldsymbol{c}$ and $\boldsymbol{d}$ were not symmetric with respect to the [110] direction. The topographical height of feature $\boldsymbol{e}$ was the lowest and slightly lower than that of Si dimer row. The adsorption positions were determined by filled- and empty-state images and cross-sectional cuts along the dimer row, to reveal that the highest protrusions (a) corresponded to benzene molecules adsorbed on a Si dimer, whereas the other protrusions $(\boldsymbol{b} \sim \boldsymbol{e}$ ) corresponded to molecules adsorbed near the bridge site between adjacent dimers.

Many adsorption configurations of benzene on $\mathrm{Si}(001)-2 \times 1$ have been proposed based on various experimental and theoretical studies. ${ }^{3-17}$ Such configurations can be categorized into two groups: those bonded to a single Si dimer and those bonded to two adjacent Si dimers. In all cases, the bonding between the carbon and $\mathrm{Si}$ atoms is believed to be of $\sigma$ character, which removes the aromaticity of the benzene ring. Two bonding configurations belong to the first group, referred to as SB and tilted configurations. In the SB configuration, the interaction with the 
(a)

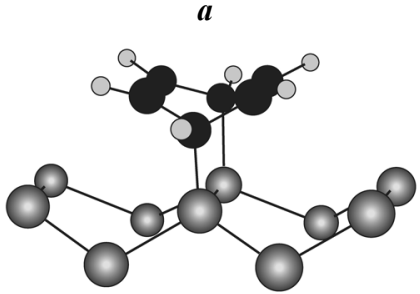

Standard butterfly (SB) (b)

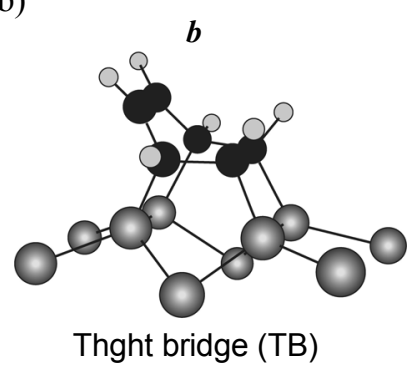

Figure 3. Schematic diagrams of the adsorption structures of benzene molecules giving rise to features $\boldsymbol{a}(\mathrm{a})$ and $\boldsymbol{b}(\mathrm{b})$. The large gradient, medium black, and small green circles represent silicon, carbon, and hydrogen atoms, respectively. Di-vacancy defects are not shown in the diagrams.

dimer occurs through the $\mathrm{C}(1)$ and $\mathrm{C}(4)$ atoms of the benzene ring; here, two double bonds are retained. The tilted configuration is formed through the interaction of two adjacent $\mathrm{C}$ atoms from the benzene ring with the dimer; the plane of the molecule is thereby tilted away from the surface. Several configurations have been proposed to describe the bonding with the adjacent Si dimers. In the pedestal form (P), the $\mathrm{C}(1), \mathrm{C}(2), \mathrm{C}(4)$, and $\mathrm{C}(5)$ atoms bond to adjacent $\mathrm{Si}$ dimers and the plane of the molecule is flat and parallel to the surface. No $\pi$ bonds remain in this case. The TB and twisted-bridge (TwB) species both involve bonding of the $\mathrm{C}(1)-\mathrm{C}(4)$ atoms with the Si dimers and retain one $\mathrm{C}=\mathrm{C}$ bond with its axis parallel to the surface. These species are azimuthally rotated (by $90^{\circ}$ ) with respect to each other. The mirror plane is perpendicular to the dimers in the case of TB and parallel to them in the case of TwB. The diagonal-bridge-butterfly (DBB) species is similar to the SB species in that only the $C(1)$ and $C(4)$ atoms of the benzene ring are involved in the surface bonding. However, the two Si atoms are in different dimers.

Taking into consideration the previous studies ${ }^{3-17}$ and the symmetry of the STM features in Figs. 1 and 2, feature $\boldsymbol{b}$ [Fig. 1(a)] was assigned to the TB configuration [Fig. 3(b)] whereas feature $\boldsymbol{a}$ was assigned to the SB configuration [Fig. 3(a)]. The different protrusion heights of features $\boldsymbol{a}$ and $\boldsymbol{b}$ was attributed to the inducement of the protrusions in the STM images by the high electron density of states. ${ }^{14,19}$ Features $\boldsymbol{c}, \boldsymbol{d}$, and $\boldsymbol{e}$ were assigned to the TwB, DBB, and P configurations, respectively. At this benzene coverage, features $\boldsymbol{a}$ and $\boldsymbol{b}$ had the most dominant population distributions, suggesting that these states are energetically and/or kinetically more favorable than the others. The population distributions of features $\boldsymbol{c} \sim \boldsymbol{e}$ were below $11 \%$.

DFT calculations ${ }^{13}$ revealed a higher adsorption energy of $1.47 \mathrm{eV}$ for TB than that of SB $(1.12 \mathrm{eV})$ on a $\mathrm{Si}(001)-2 \times 1$ surface. The fractional occurrences of features $\boldsymbol{a}$ and $\boldsymbol{b}$ in the present work were about $19 \%$ and $70 \%$, respectively, at a very low benzene coverage. These values were consistent with the calculation results. Room-temperature STM and infrared spectroscopy results for benzene on a $\mathrm{Si}(001)-2 \times 1$ surface indicated that the aromatic compound was initially chemisorbed in the $\mathrm{SB}$ form (as a metastable state) and then slowly converted to the lower-energy TB state (as the final state). ${ }^{14}$ However, the infrared experiments ${ }^{6}$ suggested that benzene is predominantly

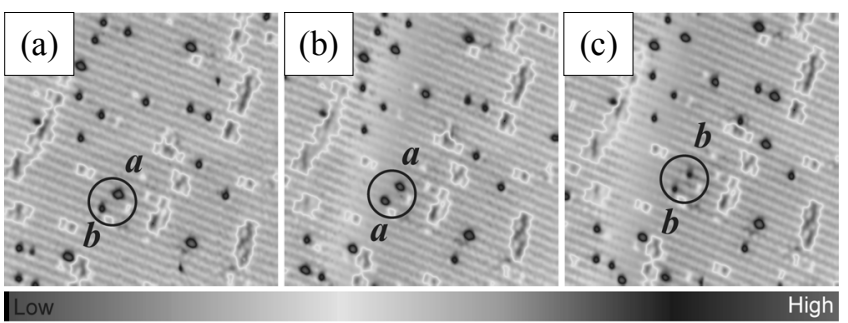

Figure 4. Conversion of the adsorption configuration of benzene on the $\mathrm{Si}(001)-2 \times \mathrm{n}$ surface. The feature $\boldsymbol{b}$ configuration in (a) is converted to feature $\boldsymbol{a}$ in (b) after scanning the same area at a tunneling current of 0.1 $\mathrm{nA}$ and a sample bias voltage of $-1.8 \mathrm{~V}$. Each feature $\boldsymbol{a}$ in (b) is converted to a feature $\boldsymbol{b}$ after scanning the area using the same tunneling conditions. The STM images were obtained at a tunneling current of 0.5 $\mathrm{nA}$ and a sample bias voltage of $-1.5 \mathrm{~V}$.

adsorbed in the SB configuration on the $\mathrm{Si}(001)-2 \times 1$ surface at room temperature, with only a small portion of the molecules, which was probably the TB configuration.

Field-induced conversion and desorption of benzene. The benzene molecules exhibited the ability to switch between features $\boldsymbol{a}$ and $\boldsymbol{b}$ when a high sample bias voltage was applied in the STM junction. Figure 4(b) displays the surface shown in Fig. 4(a) after scanning the area at a sample bias of $-1.8 \mathrm{~V}$ and a tunneling current of $0.5 \mathrm{nA}$. This scanning procedure caused feature $\boldsymbol{b}$ [found inside the circle of Fig. 4(a)] to be converted into feature $\boldsymbol{a}$ [shown in Fig. 4(b)]. When the area was scanned again using the same tunneling conditions, all of the feature $\boldsymbol{a}$ spots were converted into feature $\boldsymbol{b}$ spots [see Fig. 4(c)]. No preferential conversion direction was observed between these states at a sample bias of $-1.8 \mathrm{~V}$ and a tunneling current of 0.5 $\mathrm{nA}$. This intermolecular conversion was not, however, observed when scanning using sample bias voltages below $1.5 \mathrm{~V}$ at a tunneling current of $1.0 \mathrm{nA}$. The conversion efficiency depended only on the sample bias voltage, with negligible effect being exerted by the tunneling current $(0.1 \sim 10 \mathrm{nA})$. The voltage barrier for conversion measured at room temperature is very similar to those obtained at low surface temperatures of 80 and $115 \mathrm{~K}$, revealing the small thermal contribution to the conversion. In a previous STM study of benzene adsorbed on $\mathrm{Si}(001)-$ $2 \times 1,{ }^{14}$ the irreversible conversion from the SB to the TB configuration was observed at room temperature, with the activation barrier for the conversion being about $1 \mathrm{eV}$, which was confirmed by theoretical calculations. ${ }^{10}$

During an STM scan of a surface on which benzene molecules are adsorbed, the tip-sample field causes the benzene molecules to desorb. Figure 5(b) shows the same surface as that in Fig. 5(a) after scanning the area at a sample bias of $-2.5 \mathrm{~V}$ and a tunneling current of $0.5 \mathrm{nA}$. The application of this high sample bias voltage caused the desorption of almost all benzene molecules. In some cases, rather than being desorbed, the benzene molecules diffused across the Si surface or hopped between the tip and the surface. The remaining feature $a$, shown in Fig. 5(b), was not observed in Fig. 5(a), which suggests that this feature may have been produced by such as diffusion or hopping or conversion processes. The desorption yield (probability per electron) is measured by counting the fraction of molecules that are desorbed after scanning at a given bias voltage. This 


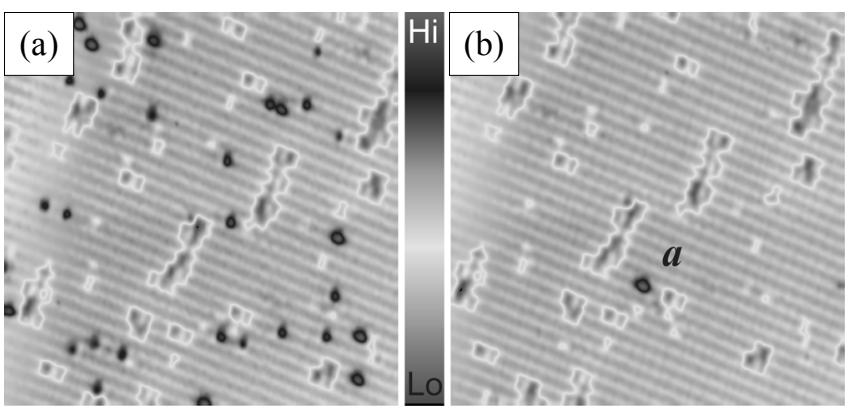

Figure 5. Desorption of benzene molecules from the $\mathrm{Si}(001)-2 \times \mathrm{n}$ surface. (a) STM image of benzene molecules adsorbed on the $\mathrm{Si}(001)-2 \times \mathrm{n}$ surface. Scanning at a sample bias voltage of $-2.5 \mathrm{~V}$ and a tunneling current of $1.0 \mathrm{nA}$ caused the desorption of most of the benzene molecules (b). The images were obtained at a sample bias voltage of $-1.5 \mathrm{~V}$ and a tunneling current of $0.5 \mathrm{nA}$.

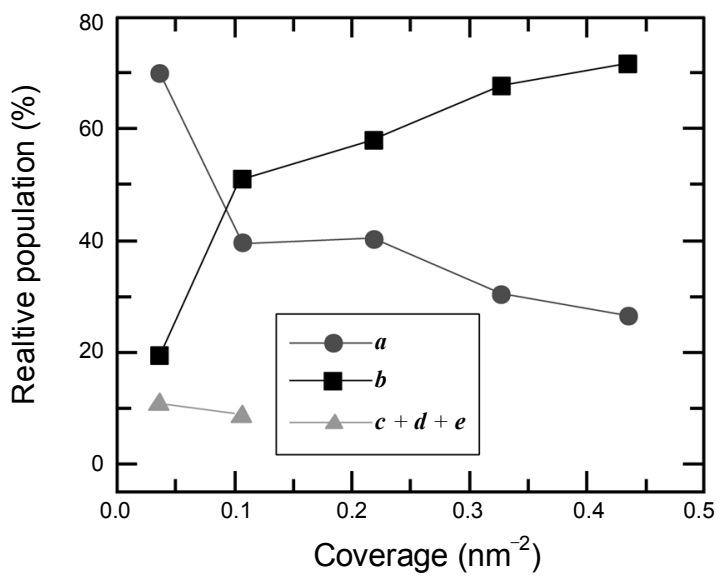

Figure 6. Relative population ratio of benzene configurations as a function of the total surface concentration. The lines connecting the points are guides for the eye.

fraction is employed to extract the desorption yield using the measured area of a single benzene molecule and the known tunneling current going through the tip. In our study, the rate of desorption depended strongly on the sample bias voltage but was less sensitive to the tunneling current $(0.1 \sim 10 \mathrm{nA})$. For example, no distinct changes in desorption rate were observed at the tunneling current of $0.1 \sim 10 \mathrm{nA}$ and a sample bias of $-2.5 \mathrm{~V}$. The desorption yield increased to $10^{-8}$ (from about $10^{-10}$ at $-2.0 \mathrm{~V}$ ) at a sample bias voltage of $-2.5 \mathrm{~V}$ and a tunneling current of $1 \mathrm{nA}$, suggesting that the desorption process may involve a field-induced lowering of the activation barrier. The desorption rate determined in the present work was lower than that obtained on $\mathrm{Si}(001)-2 \times 1\left(10^{-6}\right)$ at $22 \mathrm{~K},{ }^{15}$ indicating the stronger binding of benzene on the defected surface [Si(001)$2 \times n]$.

Coverage-dependent adsorption and desorption of benzene.

The relative population ratio between features $\boldsymbol{a}$ and $\boldsymbol{b}$ was affected by the benzene coverage. Figure 6 shows the relative population as a function of the total coverage (i.e., of the number of molecules per unit area), which was obtained by counting over 2,000 benzene molecules. At very low coverage, feature $\boldsymbol{b}$ was
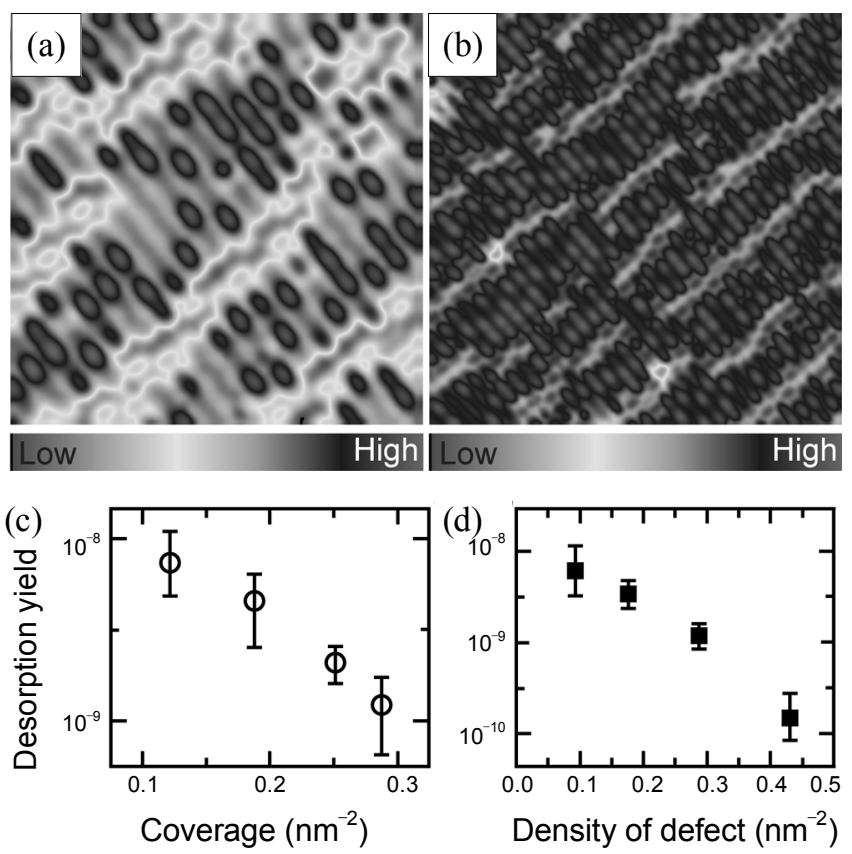

Figure 7. (a) and (b) STM topographical images of high-benzenecovered $\mathrm{Si}(001)-2 \times \mathrm{n}$ surfaces. The scan area is (a) $11.5 \times 11.5 \mathrm{~nm}^{2}$ and (b) $22.5 \times 22.5 \mathrm{~nm}^{2}$. (c) Desorption yield as a function of (c) benzene coverage and (d) density of di-vacancy defect. The yields in (c) were measured at a sample bias voltage of $2.6 \mathrm{~V}$ and a tunneling current of $1 \mathrm{nA}$ at the defect-density of $0.25 \sim 0.35 \mathrm{~nm}^{-2}$. The yields in (d) were measured at a sample bias voltage of $2.4 \mathrm{~V}$ and a tunneling current of $1 \mathrm{nA}$ at the coverage range of $0.2 \sim 0.3 \mathrm{~nm}^{-2}$.

dominant on the surface, as shown in Figs. 4 and 5. Upon increasing benzene coverage, the amount of feature $\boldsymbol{a}$ tended to increase. At high coverage, the majority of species changed to feature $\boldsymbol{a}$ (see Fig. 7). Most of the change had occurred at a coverage of approximately $0.1 \mathrm{~nm}^{-2}$.

The coverage-dependent change of the adsorption feature was similar to the previous $\operatorname{TDS}^{4,5}$ and high resolution photoemis$\operatorname{sion}^{7}$ studies, which showed two spectral peaks for the chemisorbed benzene molecules, depending on the coverage. These results demonstrated that only the adsorption species with a high desorption temperature appeared at low coverage, whereas the species with a low desorption temperature grew with increasing coverage. ${ }^{4,5}$ The adsorption species with a higher desorption temperature was attributed to chemisorbed molecules with a stronger benzene-Si interaction, which were considered to be related with the TB adsorbates.

The configuration of a chemisorbed molecule is usually dependent on its coverage on metal surfaces. ${ }^{22}$ This is explained as the result of the interplay between the substrate-molecule and molecule-molecule interactions. The configuration is determined primarily by substrate-molecule interactions at low coverage, but may change due to the increasing importance of molecule-molecule interactions at high coverage. The coverage in the present work was, however, extraordinarily low, corresponding to approximately one molecule per $10 \mathrm{~nm}^{2}$, which renders direct intermolecular interactions an unlikely driving force for the configuration change. Instead, an indirect, substrate-mediated interaction is probably involved in destabilizing the TB 
configuration. Since the structure of the Si surface dimers is maintained, an indirect interaction may take place through the strain field on the substrate. ${ }^{7}$ A subtle change of the surface strain field in the neighboring site of a benzene molecule should be introduced. Such interactions can also change the thermodynamics or kinetics of the molecular adsorption. For example, the conversion from the SB to the TB configuration may be more favorable due to steric hindrance (as shown in several STM studies performed on very low-covered $\mathrm{Si}(001)-2 \times 1$ surfaces), ${ }^{9,13}$ but the energy barrier between the two configurations could be affected by direct or indirect adsorbate-adsorbate interactions at high coverage. Direct evidence for this mechanism is still lacking, but it is clear that a molecule-molecule interaction should be required to explain the observed transition.

When nearly all Si dimers were covered with benzene molecules [very high coverage, see Fig. 7(b)], these benzene structures were very stable. No significant conversion or desorption was observed, even at sample bias voltages as high as $\pm 3 \mathrm{~V}$. This stability of the highly covered benzene surface also revealed the existence of molecule-molecule interactions. Figure 7(c) shows the desorption yield of benzene measured as a function of surface coverage. The yield decreased with increasing coverage, indicating the existence of molecule-molecule interactions.

The effect of vacancy defects on benzene desorption. Above observations on $\mathrm{Si}(001)-2 \times \mathrm{n}$ surface, which are different from those on low-defected $\mathrm{Si}(001)-2 \times 1$, indicate that many vacancy defects certainly changes the adsorption and desorption dynamics of benzene molecules. In other words, the surface potential energy is modified by the vacancy defects over a large area, which changes the adsorption and desorption energy of benzene. The higher conversion barrier between the two configurations and the lower desorption rate indicate that vacancy defects lead to a stronger binding of benzene on $\mathrm{Si}(001)-2 \times \mathrm{n}$ than on $\mathrm{Si}(001)-2 \times 1$. Figure $7(\mathrm{~d})$ shows the desorption yield measured as a function of the density of vacancy defect. The yield decreases with increasing defect density, indicating that the defects make the benzene binding much stronger on $\mathrm{Si}$. The high-coverage benzene structure along the short Si-dimer row may be stabilized by vacancy defects at both ends as well as by longrange intermolecular interactions. It was not possible to find any correlations of the adsorption fraction and desorption probability with the distance from the defects because a high sample bias voltage can induce conversion and diffusion as well as desorption.

\section{Conclusions}

The adsorption and desorption characteristics of benzene on a $\mathrm{Si}(001)-2 \times \mathrm{n}$ surface were investigated by STM. We identified five types of adsorption feature and found that the TB and SB configurations were the most dominant ones. A sample bias field induced reversible conversion between the two configurations as well as the desorption of benzene molecules from the $\mathrm{Si}(001)-2 \times \mathrm{n}$ surface. The desorption and conversion barriers of benzene on the $\mathrm{Si}(001)-2 \times \mathrm{n}$ surface are relatively higher than those on $\mathrm{Si}(001)-2 \times 1$. The desorption efficiency decreased with increasing surface coverage and vacancy defects. From the po- pulation variation of the two benzene species and the stability of the high-coverage surface, we concluded that the adsorption geometries of chemisorbed benzene vary from the TB configuration at the initial stage of coverage to the SB configuration at the quasi-saturation coverage, which we attributed to molecule-molecule interactions. Our finding of the coverage- and defect-dependent adsorption and desorption behavior of benzene may explain the discrepancy in reported findings between the microscopy and ensemble-averaged spectroscopy studies. While most of the previous STM studies investigated the very low-coverage adsorption behavior on surfaces with few defects, where the energetically stable TB structure prevails, most of the ensemble-averaged spectroscopy studies focused on the high-coverage regime with less defined defect density, where the SB structure dominates.

Acknowledgments. This work was supported by the KOSEF funded by the MOST (R11-2007-012-02001-0) and the KRF (KRF-2006-005-J00301).

\section{References}

1. (a) Waltenburg, H. N.; Yates, J. T., Jr. Chem. Rev. 1995, 95, 1589. (b) Wolkow, R. A. Annu. Rev. Phys. Chem. 1999, 50, 413. (c) Hamers, R. J.; Coulter, S. K.; Ellison, M. D.; Hovis, J. S.; Padowitz, D. F.; Schwartz, M. P.; Greenlief, C. M; Russell, J. N., Jr. Acc. Chem. Res. 2000, 33, 617.

2. (a) Hata, K.; Ishida, M.; Miyake, K.; Shigekawa, H. Appl. Phys. Lett. 1998, 73, 40. (b) Tochihara, H.; Amakusa, T.; Iwatsuki, M. Phys. Rev. B 1994, 50, 12262. (c) Koo, J.-Y.; Yi, J.-Y.; Hwang, C.; Kim, D.-H.; Lee, S.; Shin, D.-H. Phys. Rev. B 1995, 52, 17269. (d) Ukraintsev, V. A.; Yates, J. T., Jr. Surf. Sci. 1996, 346, 31. (e) Kato, K.; Ide, T.; Miura, S.; Tamura, A.; Ichinokawa, T. Surf. Sci. 1988, 194, L87. (f) Horsfield, A.; Akhmatskaya, E.; Nobes, R.; Andzelm, J.; Fitzgerald, G.; Govind, N. Phys. Rev. B 2002, 66, 085309. (g) Kolditz, B.; Roos, K. R. J. Vac. Sci. Technol. A 2007, 25, 721. (h) Yang, H. Q.; Zhu, C. X.; Gao, J. N.; Xue, Z. Q.; Pang, S. J. Surf. Sci. 1998, 412-413, 236. (i) Weber, E. R. Appl. Phys. A: Solids Surf. 1983, 30, 1. (j) Ukraintsev, V. A.; Dohnálek, Z.; Yates, J. T., Jr. Surf. Sci. 1997, 388, 132. (k) Swartzentruber, B. S.; Mo, Y.-W.; Webb, M. B.; Lagally, M. G. J. Vac. Sci. Technol. A 1989, 7, 2901. (1) Zandvliet, H. J. W. Surf. Sci. 1997, 377-379, 1.

3. (a) Li, Q.; Leung, K. T. Surf. Sci. 2001, 479, 69. (b) Kruse, P.; Wolkow, R. A. Appl. Phys. Lett. 2002, 81, 4422. (c) Hofer, W. A.; Fisher, A. J.; Lopinski, G. P.; Wolkow, R. A. Surf. Sci. 2001, 482485, 1181. (d) Jung, Y.; Gordon, M. S. J. Am. Chem. Soc. 2005, 127, 3131. (e) Nisbet, G.; Lamont, C. L. A.; Polcik, M.; Terborg, R.; Sayago, D. I.; Kittel, M.; Hoeft, J. T.; Toomes, R. L.; Woodruff, D. P. J. Phys.: Condens. Matter 2008, 20, 304206. (f) Jeong, H. D.; Ryu, S.; Lee, Y. S.; Kim, S. Surf. Sci. 1995, 344, L1226.

4. Taguchi, Y.; Fujisawa, M.; Takaoka, T.; Okada, T; Nishijima, M. J. Chem. Phys. 1991, 95, 6870.

5. Gokhale, S.; Trischberger, P.; Menzel, D.; Widdra, W.; Dröge, H.; Steinrück, H.-P.; Birkenheuer, U.; Gutdeutsch, U.; Rösch, N. J. Chem. Phys. 1998, 108, 5554

6. Kong, M. J.; Teplyakov, A. V.; Lyubovitsky, J. G.; Bent, S. F. Surf. Sci. 1998, 411, 286.

7. Kim, Y. K.; Lee, M. H.; Yeom, H. W. Phys. Rev. B 2005, 71, 115311.

8. Borovsky, B.; Krueger, M.; Ganz, E. Phys. Rev. B 1998, 57, R4269.

9. Lopinski, G. P.; Fortier, T. M.; Moffatt, D. J.; Wolkow, R. A. J. Vac. Sci. Technol. A 1998, 16, 1037.

10. (a) Lee, J.-Y.; Cho, J.-H. Phys. Rev. B 2005, 72, 235317. (b) Silvestrelli, P. L.; Ancilotto, F.; Toigo, F. Phys. Rev. B 2000, 62, 1956.

11. (a) Nagao, M.; Yamashita, Y.; Machida, S.; Hamaguchi, K.; Yasui, F.; Mukai, K.; Yoshinobu, J. Surf. Sci. 2002, 513, 413. (b) Witko- 
wski, N.; Pluchery, O.; Borensztein, Y. Phys. Rev. B 2005, 72, 075354.

12. Birkenheuer, U.; Gutdeutsch, U.; Rösch, N.; Fink, A.; Gokhale, S.; Menzel, D.; Trischberger, P.; Widdra, W. J. Chem. Phys. 1998, 108,9868 .

13. Hofer, W. A.; Fisher, A. J.; Lopinski, G. P.; Wolkow, R. A. Phys. Rev. B 2001, 63, 085314.

14. Lopinski, G. P.; Moffatt, D. J.; Wolkow, R. A. Chem. Phys. Lett. 1998, 282, 305.

15. Alavi, S.; Rousseau, R.; Patitsas, S. N.; Lopinski, G. P.; Wolkow, R. A.; Seideman, T. Phys. Rev. Lett. 2000, 85, 5372.

16. Self, K. W.; Pelzel, R. I.; Owen, J. H. G.; Yan, C.; Widdra, W.; Weinberg, W. H. J. Vac. Sci. Technol. A 1998, 16, 1031.

17. Wolkow, R. A.; Lopinski, G. P.; Moffatt, D. J. Surf. Sci. 1998, 416, L1107.

18. (a) Lu, X.; Wang, X.; Yuan, Q.; Zhang, Q. J. Am. Chem. Soc. 2003,
125, 7923. (b) Li, Z.-H.; Li, Y.-C.; Wang, W.-N.; Cao, Y.; Fan, K.-N. J. Phys. Chem. B 2004, 108, 14049.

19. (a) Hahn, J. R.; Jeong, H.; Jeong, S. J. Chem. Phys. 2005, 123 , 244702. (b) Jeong, H.; Jeong, S.; Jang, S. H.; Seo, J. M.; Hahn, J. R. J. Phys. Chem. B 2006, 110, 15912. (c) Hahn, J. R.; Jeong, H.; Jeong, S.; Jang, S. H. Jpn. J. Appl. Phys. 2006, 45, 2175.

20. (a) The scanning tunneling microscope is a modified version of that described in Stipe, B. C.; Rezaei, M. A.; Ho, W. Rev. Sci. Instrum. 1999, 70, 137. (b) Lauhon, L. J.; Ho, W. Rev. Sci. Instrum. 2001, 72, 216. (c) Hahn, J. R. Bull. Korean Chem. Soc. 2005, 26, 1071.

21. Jang, S. H.; Jeong, S.; Hahn, J. R. J. Phys. Chem. C 2007, 111, 340.

22. (a) Haq, S.; King, D. A. J. Phys. Chem. 1996, 100, 16957. (b) DiNardo, N. J.; Avouris, Ph.; Demuth, J. E. J. Chem. Phys. 1984, 81, 2169. (c) Dougherty, D. B.; Lee, J.; Yates, J. T., Jr. J. Phys. Chem. $B$ 2006, 110, 11991. 